Proyecciones

Vol. 23, No 2, pp. 97-110, August 2004.

Universidad Católica del Norte

Antofagasta - Chile

\title{
REPRESENTATION THEOREMS OF LINEAR OPERATORS ON P-ADIC FUNCTION SPACES
}

\author{
JOSÉ AGUAYO * \\ ELSA CHANDÍA \\ JACQUELINE OJEDA \\ Universidad de Concepción, Chile
}

Received January 2004. Accepted May 2004.

\begin{abstract}
Let $X$ be a 0-dimensional Hausforff topological space, $E, F$ nonarchimedean Banach spaces and $C_{b}(X, E)$ the space of all continuous $E$-valued functions on $X$ provided with two strict topologies. In this paper we show that every $F$-valued linear operator which is strictly continuous can be represented by a certain $\mathcal{L}(E, F)$-valued measure defined on the ring of all clopen subsets of $X$.
\end{abstract}

${ }^{*}$ This work is partially supported by Fondecyt No. 1020288 


\section{Introduction and notations}

By the classical Riesz Representation Theorem, a linear functional $u$ on the space of continuous real functions on a compact Hausdorff space $X$, is continuous for the topology of uniform convergence if, and only if, there exists a bounded regular Borel measure $m$ on $X$ such that $u(f)=\int f d m$. The Riesz Representation Theorem has been extended to many other spaces (see [8]) and linear operators instead of linear functional (see [4]). The relation between vector measures, linear operators and strict topologies in the classical case have been studied by several authors (see [1] [4]). Analogous situation in the non-archimedean case is studied in [5].

This paper is devoted to extend the work given in [5] for another two strict topologies. Throughout this work, $X$ will be a zero dimensional Hausdorff topological space, $\mathbf{K}$ a complete non-archimedean valued field with nontrivial valuation and $E, F$ non-archimedean Banach spaces.

We will denote by $C_{b}(X, E)$ the space of all $E$-valued bounded and continuous functions on $X$ and by $C_{r c}(X, E)$ the subspace of $C_{b}(X, E)$ of those functions whose image of $X$ are relatively compact. If $E=\mathbf{K}$, we will write $C_{b}(X)$ and $C_{r c}(X)$ respectively.

We will denote by $\beta_{\circ} X$ the Banaschewski compactification of $X$ [7] and understand by $\widehat{f}$ the unique continuous extension of $f$ to $\beta_{\circ} X$. For

$A \subset X$, we will denote by $\bar{A}^{\beta_{\circ} X}$ the closure of $A$ in $\beta_{\circ} X$ and by $\mathcal{X}_{A}$ the $\mathbf{K}$-valued characteristic function of $A$. For an $E$-valued function $f$ on $X$ and $A \subset X$, we will denote

$$
\|f\|_{A}=\sup _{x \in A}\|f(x)\|, \quad\|f\|_{X}=\|f\|
$$

Let $\mathcal{S}(X)$ be the collection of all clopen subsets of $X$. An $\mathcal{L}(E, F)-$ valued set function $m$ on $\mathcal{S}(X)$ is said to be a measure if:

1. $m$ is finitely additive

2. The set $m(\mathcal{S}(X))$ is bounded in $\mathcal{L}(E, F)$.

We will denote by $M(X, \mathcal{L}(E, F))$ the space of all these measures. For $m \in M(X, \mathcal{L}(E, F))$ and $A \in \mathcal{S}(X)$, we define

$$
\|m\|(A)=\sup \left\{\|m(B) e\|_{F}: B \subset A, B \in \mathcal{S}(X),\|e\|_{E} \leq 1\right\} .
$$

In order to introduce strict topologies, we will denote by $\Omega$ the collection of all compact subsets of $\beta_{\circ} X \backslash X$ and by $\Omega_{u}$ the collection of $Q \in \Omega$ such 
that $Q$ admits a clopen partition $\left\{U_{\alpha}\right\}_{\alpha \in I}$ of $X$ such that $\bar{U}_{\alpha}^{\beta_{\circ} X} \cap Q=\emptyset$ for all $\alpha \in I$.

The strict topology $\beta\left(\beta_{u}\right)$ on $C_{b}(X, E)$ is the inductive limit of the locally convex topologies $\beta_{Q}$, where $\beta_{Q}$ is generated by the family of seminorms $f \mapsto\|g f\|$, where $g \in C_{Q}=\left\{g \in C_{r c}(X): \widehat{g}_{\mid Q} \equiv 0\right\}$ and $Q \in \Omega\left(\Omega_{u}\right)$ (see $[5],[2,3])$.

Next, we will define the integrability of an $E$-valued function $f$ on $X$ with respect to a $m \in M(X, \mathcal{L}(E, F))$. For $A \in \mathcal{S}(X), A \neq \emptyset$, let $\mathcal{D}_{A}$ denote the family of all $\alpha=\left\{A_{1}, \cdots, A_{n} ; x_{1}, \cdots, x_{n}\right\}$, where $\left\{A_{1}, \cdots, A_{n}\right\}$ is a clopen partition of $A$ and $x_{i} \in A_{i}$. We will introduce the following relation: $\alpha_{1} \geq \alpha_{2}$ iff the partition of $A$ in $\alpha_{1}$ is a refinement of the partition of $A$ in $\alpha_{2}$. We will denote by $\Omega_{A}$ the collection of all these $\alpha . \Omega_{A}$ will become to be a directed set. For $f, m$ and $\alpha \in \Omega_{A}, \alpha=\left\{A_{1}, \cdots, A_{n} ; x_{1}, \cdots, x_{n}\right\}$, we will define

$$
\varpi(f, m)=\sum_{n=1}^{n} m\left(A_{i}\right)\left(f\left(x_{i}\right)\right) .
$$

Note that $\varpi(f, m) \in F$.

We will say that $f$ is $m$-integrable over $A$ if $\lim _{\alpha} \varpi(f, m)$ exists; in such a case, we will denote this limit by

$$
\int_{A} f d m=\lim _{\alpha} \varpi(f, m) .
$$

If $A=\emptyset$, then we will define $\int_{\emptyset} f d m=0$. For $A=X$, we will simply write $\int f d m$. It is easy to see that if $f$ is $m$-integrable over $X$, then $f$ is $m$-integrable over every $A \in \mathcal{S}(X)$.

We will present the following very well-known technical result.

Lemma 1. Let $\varepsilon>0$ and $f \in C_{r c}(X, E)$. Then, there exist disjoint clopen sets $A_{1}, A_{2}, \ldots, A_{n}$ covering $X$ and elements $e_{1}, e_{2}, \ldots, e_{n}$ of $E$ such that

$$
\left\|f-\sum_{i=1}^{n} \mathcal{X}_{A_{i}} e_{i}\right\| \leq \varepsilon,
$$

where $\mathcal{X}_{A_{i}} e_{i}(x)=e_{i}$, if $x \in A_{i}$ and the null element $\theta$ of $E$ otherwise.

\section{The space $\mathcal{L}\left(C_{r c}(X, E), F\right)$.}

In this section we will study the relation between measure theory and $F$-valued continuous linear operators on $C_{r c}(X, E)$. We will denote by $\mathcal{L}\left(C_{r c}(X, E), F\right)$ the space of all these operators. 
Theorem 2 : If $f \in C_{r c}(X, E)$ and $m \in M(X, \mathcal{L}(E, F))$, then $f$ is $m$ integrable over $A$, for each $A \in \mathcal{S}(X)$.

Proof. Without loss of generality, we can assume that $A=X$ and $\|m\|(X) \leq 1$. Let $\mu \in \mathbf{K}$, with $0<|\mu| \leq 1$, and $\varepsilon>0$. We take $\nu \in \mathbf{K}$ such that $0<|\nu|<|\mu| \varepsilon$, Since $f \in C_{r c}(X, E)$, there exists a finite clopen partition $\left\{A_{1}, A_{2}, \ldots, A_{n}\right\}$ of $X$ and finite subset $\left\{e_{1}, e_{2}, \ldots, e_{n}\right\}$ of $E$ such that

$$
\left\|f(x)-e_{i}\right\|_{E} \leq|\nu|, \forall x \in A_{i}
$$

Choose $x_{i} \in A_{i}$ and consider $\alpha_{\circ}=\left\{A_{1}, A_{2}, \ldots, A_{n} ; x_{1}, x_{2}, \ldots, x_{n}\right\}$. Take $\alpha=\left\{B_{1}, B_{2}, \ldots, B_{k} ; y_{1}, y_{2}, \ldots, y_{k}\right\}$ such that $\alpha \geq \alpha_{\circ}$. Then, for $B_{j} \subset A_{i}$, we have

$$
\begin{aligned}
\left\|f\left(y_{j}\right)-f\left(x_{i}\right)\right\|_{E} & =\left\|f\left(y_{j}\right)-e_{i}+e_{i}-f\left(x_{i}\right)\right\|_{E} \\
& \leq \max \left\{\left\|f\left(y_{j}\right)-e_{i}\right\|_{E},\left\|e_{i}-f\left(x_{i}\right)\right\|_{E}\right\} \\
& \leq|\nu|
\end{aligned}
$$

Thus, if $\lambda=\nu^{-1} \mu$, then

$$
\left\|\lambda\left[f\left(y_{j}\right)-f\left(x_{i}\right)\right]\right\|_{E} \leq|\lambda||\nu| \leq|\mu| \leq 1
$$

and then

$$
\left\|m(A)\left(\lambda\left[f\left(y_{j}\right)-f\left(x_{i}\right)\right]\right)\right\| \leq 1
$$

which implies

$$
\left\|m(A)\left(f\left(y_{j}\right)-f\left(x_{i}\right)\right)\right\|_{F} \leq|\nu||\mu|^{-1} \leq \varepsilon
$$

Therefore,

$$
\begin{aligned}
\left\|\omega_{\alpha}(f, m)-\omega_{\alpha_{\circ}}(f, m)\right\|_{F}= & \left\|\sum_{j=1}^{N} m\left(A_{j}\right) f\left(y_{i}\right)-\sum_{i=1}^{k} m\left(B_{i}\right) f\left(x_{i}\right)\right\|_{F} \\
& \leq \max _{1 \leq j \leq N}\left\|m\left(A_{j}\right)\left(f\left(y_{i}\right)-f\left(x_{i}\right)\right)\right\|_{F}
\end{aligned}
$$

Now, if $\alpha_{1}, \alpha_{2} \geq \alpha_{\circ}$, then

$\left\|\omega_{\alpha_{1}}(f, m)-\omega_{\alpha_{2}}(f, m)\right\|_{F}=\left\|\omega_{\alpha_{1}}(f, m)-\omega_{\alpha_{\circ}}(f, m)+\omega_{\alpha_{\circ}}(f, m)-\omega_{\alpha_{2}}(f, m)\right\|_{F}$ $\leq \max \left\{\left\|\omega_{\alpha_{1}}(f, m)-\omega_{\alpha_{0}}(f, m)\right\|_{F},\left\|\omega_{\alpha_{0}}(f, m)-\omega_{\alpha_{2}}(f, m)\right\|_{F}\right\} \leq \varepsilon$.

This proves that the net $\left\{\omega_{\alpha}(f, m)\right\}$ is Cauchy in $F$ and hence convergent, since $F$ is complete.

Lemma 3 : Let $f: X \rightarrow E$ be a $m$-integrable function over $A \in \mathcal{S}(X)$. 
1. If the valuation of $\mathbf{K}$ is dense or the valuation is discrete and $\|E\| \subset|\mathbf{K}|$, then

$$
\left\|\int_{A} f d m\right\| \leq\|f\|_{A}\|m\|(A)
$$

2. If the valuation of $\mathbf{K}$ is discrete and if $\rho>1$ is the generator of $|\mathbf{K}| \backslash\{0\}$, then

$$
\left\|\int_{A} f d m\right\| \leq \rho\|f\|_{A}\|m\|(A) .
$$

Proof. 1.) We can assume that $0<\|f\|_{A}<\infty$, since otherwise, the statement is trivial. Under the denseness conditions, for each $\varepsilon>0$, there exists $\lambda \in \mathbf{K}$ such that

$$
\|f\|_{A} \leq \lambda<\|f\|_{A}+\varepsilon
$$

Since $\left\|\lambda^{-1} f(x)\right\| \leq 1, \forall x \in A$, we have

$$
\begin{aligned}
|\lambda|^{-1}\left\|\omega_{\alpha}(f, m)\right\| & =\left\|\omega_{\alpha}\left(\lambda^{-1} f, m\right)\right\| \\
& =\left\|\sum_{i=1}^{n} m\left(A_{i}\right) \lambda^{-1} f\left(x_{i}\right)\right\| \\
& \leq\|m\|(A)
\end{aligned}
$$

for each $\alpha \in \Omega_{A}$. It follows that

$$
\left\|\int_{A} f d m\right\| \leq\|f\|_{A}\|m\|(A)
$$

2.) If we consider the norm $\|e\|^{*}=\inf \{|\lambda|: \lambda \in \mathbf{K},|\lambda| \geq\|e\|\}$ on $E$, then $\|E\|^{*} \subset|\mathbf{K}|$ and $\|\cdot\|^{*} \leq \rho\|\cdot\|$. Therefore, from 1.), we have

$$
\left\|\int_{A} f d m\right\| \leq\|f\|_{A}^{*}\|m\|^{*}(A) \leq \rho\|f\|_{A}\|m\|(A) .
$$

Remark 4 : The previous lemma proves that if $m \in M(X, \mathcal{L}(E, F))$, then the linear operator $T_{m}: C_{r c}(X, E) \rightarrow F$ defined by $T_{m}(f)=\int f d m$ is a $\mathcal{T}_{u}$-continuous linear operator, where $\mathcal{T}_{u}$ denotes the uniform convergence topology on $C_{r c}(X, E)$.

Theorem 5 : If $T: C_{r c}(X, E) \rightarrow F$ is a $\mathcal{T}_{u}$-continuous linear operator, then there exists $m \in M(X, \mathcal{L}(E, F))$ such that $T=T_{m}$. 
Proof. For each $A \in \mathcal{S}(X)$, we define

$$
\begin{aligned}
& m(A): \quad E \quad \rightarrow F \\
& e \mapsto m(A) e=T\left(\mathcal{X}_{A} e\right)
\end{aligned}
$$

Since $T$ is bounded, with bound $M>0$, we have

$$
\begin{aligned}
\|m(A) e\| & =\left\|T\left(\mathcal{X}_{A} e\right)\right\| \\
& \leq M\left\|\mathcal{X}_{A} e\right\| \\
& \leq M\|e\|
\end{aligned}
$$

that is, $m(A) \in \mathcal{L}(E, F)$. We claim that the set-function

$$
\begin{aligned}
m: \mathcal{S}(X) & \rightarrow \mathcal{L}(E, F) \\
A & \mapsto m(A)
\end{aligned}
$$

is a measure. In fact, trivially $m$ is well-define and finitely additive. To prove that $\{m(A): A \in \mathcal{S}(X)\}$ is equicontinuous, take $\varepsilon>0$ and choose $\delta=\varepsilon / M$; hence,

$$
(\forall A \in \mathcal{S}(X))(\|e\| \leq \delta \Rightarrow\|m(A) e\| \leq M\|e\| \leq M \delta=\varepsilon) .
$$

Finally, we claim that $T=T_{m}$. In fact, if $f=\sum_{i=1}^{n} \mathcal{X}_{A_{i}} e_{i}$, then it is immediately to prove $T_{m}(f)=T(f)$. On the other hand, by the facts that $\left\langle\left\{\mathcal{X}_{A} e: e \in E, A \in \mathcal{S}(X)\right\}\right\rangle$ is $\mathcal{T}_{u}$-dense in $C_{r c}(X, E)$ (see Lemma 1) and both $T_{m}$ and $T$ are $\mathcal{T}_{u}$-continuous, we get $T_{m}(f)=T(f), \forall f \in C_{r c}(X, E)$.

Corollary 6 : The mapping

$$
\begin{aligned}
\Psi: \quad M(X, \mathcal{L}(E ; F)) & \rightarrow \mathcal{L}\left(C_{r c}(X, E), F\right) \\
m & \longmapsto \Psi(m)=T_{m}
\end{aligned}
$$

is an algebraic isomorphism.

\section{3. $\tau$ and u-additive measures.}

This section will devote to study certain class of members of $M(X, \mathcal{L}(E, F))$ and study the behavior of the associated $F$-valued continuous linear operators given in the previous section.

Definition 7 : Let $m \in M(X, \mathcal{L}(E, F))$. We will say that 
1. $m$ is $\tau$-additive if for each decreasing net $\left\{A_{\alpha}\right\}_{\alpha \in I}$ in $\mathcal{S}(X)$ such that $A_{\alpha} \downarrow \emptyset$, we have

$$
\|m\|\left(A_{\alpha}\right) \rightarrow 0
$$

2. $m$ is $u$-additive if for each clopen partition $\left\{U_{\alpha}\right\}_{\alpha \in I}$ of $X$, we have

$$
\|m\|\left(X \backslash \bigcup_{j \in J} U_{j}\right) \rightarrow 0
$$

where the limit has to be taken over the directed set of all finite subsets $J \subset I$.

Proposition 8 : $M_{\tau}(X, \mathcal{L}(E, F)) \subseteq M_{u}(X, \mathcal{L}(E, F))$

Proof. Let $m \in M_{\tau}(X, L(E, F))$ and $\left(U_{\alpha}\right)_{\alpha \in I}$ be a clopen partition of $X$. For any finite subset $J$ of $I$, we define the decreasing net $\left\{A_{J}\right\}_{J}$, where $A_{J}=X \backslash \bigcup_{j \in J} U_{j}$. Now, since $\left(U_{\alpha}\right)_{\alpha \in I}$ is a clopen partition of $X$, we have that $A_{j} \downarrow \emptyset$; therefore, $\|m\|\left(A_{J}\right)=\|m\|\left(X \backslash \bigcup_{j \in J} \mathcal{U}_{j}\right) \rightarrow 0$. Therefore, $m \in M_{u}(X, \mathcal{L}(E, F))$.

In the previous section we proved that if $f \in C_{r c}(X, E)$ and $m \in$ $M(X, \mathcal{L}(E, F))$, then $f$ is $m$-integrable over any $A \in \mathcal{S}(X)$. The next theorem will extend this result.

Theorem 9 : If $f \in C_{b}(X, E)$ and $m \in M_{u}(X, \mathcal{L}(E, F))$, then $f$ is $m$ integrable over $A$, for each $A \in \mathcal{S}(X)$.

Proof. Without loss of generality, we can assume that $\|f\| \leq 1$, and $\|m\|(X) \leq 1$. For a given $\varepsilon>0$, we define the following equivalence relation

$$
x \sim y \Leftrightarrow\|f(x)-f(y)\| \leq \varepsilon
$$

Note that the corresponding equivalent classes $\left\{A_{i}\right\}_{i \in I}$ form a clopen partition of $X$. Let us choose $x_{i} \in A_{i}$ and define $g=\sum_{i \in I} \mathcal{X}_{A_{i}} f\left(x_{i}\right)$. Clearly, $g \in C_{b}(X, E)$. For a finite subsets $J$ of $I$, we denote by $B_{J}=X \backslash \bigcup_{j \in J} \cup A_{j}$. 
Each $B_{J}$ is clopen and $B_{J} \downarrow \emptyset$. Since $m$ is $u$-additive, we have that there exists a finite subset $J_{\circ}$ of $I$ such that if $J$ is another finite subset of $I$ with $J_{\circ} \subseteq J$, then $\|m\|\left(B_{J}\right) \leq \varepsilon$. For such a $J$, we define the following functions

$$
\begin{gathered}
g_{J}=\sum_{j \in J} \mathcal{X}_{A_{j}} f\left(x_{j}\right) \\
h_{J}=g-g_{J}=\sum_{i \notin J} \mathcal{X}_{A_{i}} f\left(x_{i}\right)
\end{gathered}
$$

Let us consider the finite clopen partition $\left\{A_{j}: j \in J\right\} \cup\left\{B_{J}\right\}$ of $X$ and take $\left\{D_{1}, \ldots D_{n}\right\}$ a refinement of $\left\{A_{j}: j \in J\right\} \cup\left\{B_{J}\right\}_{j}$. If we choose $y_{i} \in D_{i}$, then $g_{J}\left(y_{i}\right)=f\left(x_{j}\right)$ and $h_{J}\left(y_{i}\right)=0$, if $D_{i} \subseteq A_{j}$ for some $j \in J$, or $g_{J}\left(y_{i}\right)=0$ and $h_{J}\left(y_{i}\right)=f\left(x_{j}\right)$, for some $j \notin J$, if $D_{i} \subset B_{J}$. Therefore, if we denote by $\alpha=\left\{D_{1}, D_{2}, \ldots D_{n} ; y_{1}, \cdots, y_{n}\right\}$ and $\alpha_{\circ}=\left\{A_{1}, \ldots A_{m}, B_{J} ; x_{1}, \cdots x_{m}, x_{J}\right\}$, then

$$
\begin{aligned}
& \left\|\varpi_{\alpha}(h, m)\right\|=\left\|\sum_{D_{K} \subseteq A_{j}} m\left(D_{k}\right)\left(h_{J}\left(y_{k}\right)\right)+\sum_{D_{k} \subset B_{J}} m\left(D_{k}\right)\left(f\left(x_{i}\right)\right)\right\| \\
& =\left\|\sum_{D_{k} \subset B_{J}} m\left(D_{k}\right)\left(f\left(x_{i}\right)\right)\right\| \\
& \leq \max \left\{\left\|m\left(D_{k}\right)\left(f\left(x_{i}\right)\right)\right\|: D_{k} \subseteq B_{J}\right\} \\
& \leq\|m\|\left(B_{J}\right) \leq \varepsilon .
\end{aligned}
$$

On the other hand,

$$
\begin{aligned}
\varpi_{\alpha}\left(g_{J}, m\right) & =\sum_{D_{k} \subset A_{j}} m\left(D_{k}\right)\left(f\left(x_{j}\right)\right) \\
& =\sum_{j \in J} m\left(A_{j}\right)\left(f\left(x_{j}\right)\right)
\end{aligned}
$$

Therefore,

$$
\begin{aligned}
& \left\|\varpi_{\alpha}(g, m)-\sum_{j \in J} m\left(A_{j}\right)\left(f\left(x_{j}\right)\right)\right\| \\
& =\left\|\sum_{k=1}^{n} m\left(D_{k}\right)\left(g\left(y_{k}\right)\right)-\sum_{k=1}^{n} m\left(D_{k}\right)\left(g_{J}\left(y_{k}\right)\right)\right\| \\
& =\left\|\varpi_{\alpha}\left(g-g_{J}, m\right)\right\| \\
& =\left\|\varpi_{\alpha}\left(h_{J}, m\right)\right\| \leq \varepsilon .
\end{aligned}
$$


Also, since $\left\|f\left(y_{k}\right)-f\left(x_{i}\right)\right\| \leq \varepsilon$,

$$
\begin{aligned}
& \begin{aligned}
\left\|\varpi_{\alpha}(f-g, m)\right\| & =\left\|\sum_{k=1}^{n} m\left(D_{k}\right)\left(f\left(y_{k}\right)-f\left(x_{i}\right)\right)\right\| \\
& \leq \max \left\{\left\|m\left(D_{k}\right)\left(f\left(y_{k}\right)-f\left(x_{i}\right)\right)\right\| ; k=1, \ldots, n\right\} \\
& \leq \max \left\{\left\|m\left(D_{k}\right)\right\|\left\|f\left(y_{k}\right)-f\left(x_{i}\right)\right\| ; k=1, \ldots, n\right\} \\
& \leq \varepsilon \max \left\{\left\|m\left(D_{k}\right)\right\| ; k=1, \ldots, n\right\}
\end{aligned} \\
& \quad \text { Thus, }\left\|\varpi_{\alpha}(f, m)-\sum_{j \in J} m\left(A_{j}\right)\left(f\left(x_{j}\right)\right)\right\| \\
& =\left\|\varpi_{\alpha}(f, m)-\varpi_{\alpha}(g, m)+\varpi_{\alpha}(g, m)-\sum_{j \in J} m\left(A_{j}\right)\left(f\left(x_{j}\right)\right)\right\| \\
& =\max \left\{\left\|\varpi_{\alpha}(f, m)-\varpi_{\alpha}(g, m)\right\|,\left\|\varpi_{\alpha}(g, m)-\sum_{j \in J} m\left(A_{j}\right)\left(f\left(x_{j}\right)\right)\right\|\right\} \\
& \leq \varepsilon
\end{aligned}
$$

Now, if $\left\{G_{1}, G_{2}, \ldots, G_{s}\right\}$ is another refinement of $\left\{A_{j}: j \in J\right\} \cup B_{J}$ and $\beta=\left\{G_{1}, G_{2}, \ldots, G_{s} ; z_{1}, \cdots z_{s}\right\}$, then

$$
\begin{aligned}
& \left\|\varpi_{\alpha}(f, m)-\varpi_{\beta}(f, m)\right\| \\
& \quad=\left\|\varpi_{\alpha}(f, m)-\sum m\left(A_{j}\right)\left(f\left(x_{j}\right)\right)+\sum m\left(A_{j}\right)\left(f\left(x_{j}\right)\right)-\varpi_{\beta}(f, m)\right\| \\
& \quad=\max \left\{\left\|\varpi_{\alpha}(f, m)-\sum m\left(A_{j}\right)\left(f\left(x_{j}\right)\right)\right\|,\left\|\sum m\left(A_{j}\right)\left(f\left(x_{j}\right)\right)-\varpi_{\beta}(f, m)\right\|\right\} \\
& \quad \leq \varepsilon
\end{aligned}
$$

Therefore, $\left\{\omega_{\alpha}(f, m)\right\}$ is a Cauchy net in $F$, which is convergent since $F$ is Banach. This proves that $f$ is $m$-integrable over $A$.

Remark 10 : From Prop. 8, if $m$ is $\tau$-additive measure and $f \in$ $C_{b}(X, E)$, then $f$ is $m$-integrable over $A$. In [6], it has been proved that $M_{t}(X, \mathcal{L}(E, F))$ and the space of all $F$-valued and $\beta_{\circ}$-continuous linear operators on $C_{b}(X, E)$ are algebraically isomorphic. The next theorems will show similar results for $M_{\tau}(X, \mathcal{L}(E, F))$ and $M_{u}(X, \mathcal{L}(E, F))$.

Theorem 11 : If $T \in \mathcal{L}\left(C_{b}(X, E), F\right)$, then the following statements are equivalent: 
1. $T$ is $\beta$-continuous.

2. The associated measure $m$ is $\tau$-additive.

Proof. 1.) $\Rightarrow 2$.) Let $\left\{A_{\alpha}\right\}_{\alpha \in I}$ be a net of clopen subsets of $X$ such that $A_{\alpha} \downarrow \emptyset$. By the continuity of $T$,

$$
\mathcal{W}=\left\{f \in C_{b}(X, E):\|T(f)\| \leq 1\right\}
$$

is a $\beta$-neighborhood of 0 , and by the definition of $\beta, \mathcal{W}$ is a $\beta_{K}$-neighborhood of 0 , for all $K \in \Omega$. Now, since ${\overline{A_{\alpha}}}^{\beta_{\circ} X} \downarrow Q \in \Omega$, there exists $h \in C_{Q}(X)$ such that

$$
\mathcal{U}=\left\{f \in C_{b}(X, E):\|f\|_{h} \leq 1\right\} \subset \mathcal{W} .
$$

For a given $\varepsilon>0$, we choose $\mu \in \mathbf{K}$, with $0<|\mu|<\varepsilon$ and define

$$
G=\left\{x \in \beta_{o} X:|\widehat{h}(x)| \leq|\mu|\right\} .
$$

Note that $Q \subset G$; hence, there exists $\alpha_{\circ} \in I$ such that $\overline{A_{\alpha_{\circ}}} \beta_{\circ} X \subset G$. Now, if $\alpha \geq \alpha_{\circ}$ and $\|e\| \leq 1$, then for any $A \subset A_{\alpha}, A \in \mathcal{S}(X)$,

$$
\begin{aligned}
& \left\|\mu^{-1} \mathcal{X}_{A} e\right\|_{h}=\sup _{x \in A} \\
& \leq|\mu|^{-1} \sup _{x \in A}|h(x)| \\
& \leq|\mu|^{-1}|\mu|=1,
\end{aligned}
$$

that is, $\mu^{-1} \mathcal{X}_{A} e \in \mathcal{U}$, and then $\left\|T\left(\mu^{-1} \mathcal{X}_{A} e\right)\right\| \leq 1$ or equivalently

$$
\|m(A) e\| \leq|\mu| \leq \varepsilon
$$

Therefore, since $e$ and $A$ are arbitrary, we have $\|m\|(A) \leq \varepsilon$.

therefore $m \in M_{\tau}(X, \mathcal{L}(E, F))$.

2.) $\Rightarrow$ 1.) We will prove that

$$
\mathcal{W}=\left\{f \in C_{b}(X, E):\|T f\| \leq 1\right\}
$$

is a $\beta$-neighborhood of 0 . Let us take $d>0$ and choose $\lambda, \gamma \in \mathbf{K}$ such that $|\lambda| \geq d$ and $|\gamma|\|m\|(X) \leq 1$. Let $Q \in \Omega$; hence, there exists a net $\left\{B_{\alpha}\right\}_{\alpha \in I}$ 
in $\beta_{\circ} X$ with $B_{\alpha} \downarrow Q$. Now, if $A_{\alpha}=B_{\alpha} \cap X$, then $\left\{A_{\alpha}\right\}_{\alpha \in I}$ is net of clopen subsets of $X$ with $A_{\alpha} \downarrow \emptyset$. Thus, there exists $\alpha_{\circ}$ such that

$$
\alpha \geq \alpha_{\circ} \Rightarrow\|m\|\left(A_{\alpha}\right) \leq|\lambda|^{-1} .
$$

The clopen subset $D=X \backslash A_{\alpha_{\circ}}$ of $X$ satisfies $\bar{D}^{\beta_{o} X} \cap Q=\emptyset$. We claim that the $\beta_{Q}$-neighborhood of 0

$$
\mathcal{U}=\left\{f \in C_{b}(X, E):\|f\| \leq d \wedge\|f\|_{D} \leq|\gamma|\right\}
$$

is contained in $\mathcal{W}$. In fact, if $f \in \mathcal{U}$, then

$$
\begin{aligned}
\|T(f)\| & =\left\|\int f d m\right\| \\
& =\left\|\int_{D} f d m+\int_{A_{\alpha_{\circ}}} f d m\right\| .
\end{aligned}
$$

Now,

$$
\begin{aligned}
\left\|\int_{A_{\alpha_{\circ}}} f d m\right\| & \leq\|f\|\|m\|\left(A_{\alpha_{\circ}}\right) \\
& \leq|\lambda||\lambda|^{-1}=1
\end{aligned}
$$

and

$$
\begin{aligned}
\left\|\int_{D} f d m\right\| & \leq\|f\|_{D}\|m\|(D) \\
& \leq|\gamma|\|m\|(D) \\
& \leq|\gamma|\|m\|(X) \\
& \leq|\gamma||\gamma|^{-1}=1
\end{aligned}
$$

therefore $f \in \mathcal{W}$.

Theorem 12 : If $m \in M_{\tau}(X, L(E, F))$, then the linear operator

$$
\begin{aligned}
T_{m}: \quad C_{b}(X, E) & \rightarrow F \\
f & \mapsto T_{m}(f)=\int f d m
\end{aligned}
$$

is $\beta$-continuous.

Proof. The same arguments used in the previous theorem proves this statement and then we omit the proof.

Theorem $13: M_{\tau}(X, \mathcal{L}(E, F))$ and the space of all $F$-valued and $\beta$ continuous linear operators on $C_{b}(X, E)$ are algebraically isomorphic. 
Proof. In order to prove this theorem, we need to prove that the linear map

$$
\begin{aligned}
\Psi: \quad M_{\tau}(X, \mathcal{L}(E, F)) & \left.\rightarrow \mathcal{L}_{\beta}\left(C_{b}(X, E), F\right)\right) \\
m & \mapsto \Psi(m)=T_{m}
\end{aligned}
$$

is an algebraic isomorphism, where $\left.\mathcal{L}_{\beta}\left(C_{b}(X, E), F\right)\right)$ denotes the space of all $\beta$-continuous linear operators.

It is easy to see that the map $\Psi$ is linear and one to one. To prove that $\Psi$ is onto, take $T: C_{b}(X, E) \rightarrow F$ and prove that $T=T_{m}$ for the associated measures $m$.

By Th. $11, m \in M_{\tau}(X, \mathcal{L}(E, F))$ and $T=T_{m}$ on $C_{b}(X, E)$ follows from the $\beta$-denseness of $C_{r c}(X, E)$ in $C_{b}(X, E)$ and the $\beta$-continuity of both $T$ and $T_{m}$.

Theorem 14: If $m \in M_{u}(X, \mathcal{L}(E, F))$, then $T_{m}$ is $\beta_{u}$-continuous.

Proof. Let $\mathcal{W}=\left\{f \in C_{b}(X, E):\left\|T_{m}(f)\right\| \leq 1\right\}$ and $Q \in \Omega_{u}$. There exists a clopen partition $\left\{A_{i}\right\}_{i \in I}$ of $X$ such that $Q \cap{\overline{A_{i}}}^{\beta_{\circ} X}=\emptyset, \forall i \in I$.

For any finite subset $J$ of $I$, we define $B_{J}=X \backslash \bigcup_{i \in J} A_{i}$. Since $m \in$ $M_{u}(X, \mathcal{L}(E, F))$, there exists a finite subset $J_{\circ}$ of $I$ such that for a given $\delta>0$, we have

$$
\|m\|\left(B_{J}\right) \leq \delta^{-1},
$$

for any finite subset $J$ of $I$ with $J_{\circ} \subset J$. If $B=i \in J_{\circ} \cup A_{i}$, then $B$ is clopen in $X$ and $\bar{B}^{\beta \circ X} \cap Q=\emptyset$.

We claim that $\mathcal{U}=\left\{f \in C_{b}(X, E):\|f\| \leq \delta,\|f\|_{B} \leq 1\right\} \subset \mathcal{W}$. In fact, if $f \in C_{b}(X, E)$, then

$$
\left\|\int_{B} f d m\right\| \leq\|f\|_{B}\|m\|(B) \leq\|f\|_{B}\|m\|(X) \leq 1 .
$$

On the other hand,

$$
\left\|\int_{X \backslash B} f d m\right\| \leq\|f\|\|m\|\left(B_{J}\right) \leq \delta \delta^{-1}=1
$$

Thus,

$$
\left\|T_{m}(f)\right\| \leq \max \left\{\left|\int_{B} f d m\right|,\left|\int_{X \backslash B} f d m\right|\right\} \leq 1
$$

Therefore, since $Q \in \Omega_{u}$ is arbitrary, the continuity of $T_{m}$ follows.

Theorem 15: $M_{u}(X, L(E, F))$ and the space of all $F$-valued and $\beta_{u^{-}}$ continuous linear operators on $C_{b}(X, E)$ are algebraically isomorphic. 
Proof. As before, we only have to prove that if $T$ is a $F$-valued and $\beta_{u}$-continuous linear operators on $C_{b}(X, E)$, then $T=T_{m}$.

Let $T$ be given and $m$ be the associated measure, that is, $T(f)=\int f d m$, $f \in C_{r c}(X, E)$. We need to prove first that $m$ is $u$-additive.

Let $\left\{A_{i}\right\}_{i \in I}$ be a clopen partition of $X$ and $Q=\beta_{\circ} X \backslash \bigcup_{i \in I}{\overline{A_{i}}}^{\beta_{o} X}$. Clearly, $Q \in \Omega_{u}$ and then $T$ is $\beta_{Q}$-continuous. Thus, there exists $h \in C_{Q}$ such that

$$
\mathcal{V}_{1}=\left\{f \in C_{b}(X, E):\|f\|_{h} \leq 1\right\} \subset \mathcal{W}=\left\{f \in C_{b}(X, E):\|T(f)\| \leq 1\right\} .
$$

Take $\varepsilon>0$ and choose $\lambda \in \mathbf{K}$ with $0<|\lambda| \leq \varepsilon$. The set

$$
A=\left\{x \in \beta_{\circ} X:|\widehat{h}(x)| \leq|\lambda|\right\}
$$

is clopen in $\beta_{\circ} X, Q \subset \beta_{\circ}$ and $X \backslash A \subset \bigcup_{i \in I}{\overline{A_{i}}}^{\beta_{\circ} X}$. Now, by the compactness of $\beta_{o} X \backslash A$, there exists a finite subset $J_{\circ}$ of $I$ such that $\beta_{\circ} X \backslash A \subset \bigcup_{i \in J_{\circ}} \cup{\overline{A_{i}}}^{\beta_{\circ} X}$.

Takes a finite subset $J \subset I$ with $J_{\circ} \subset J$, and take any clopen $B$ of $X$ contained in $X \backslash \bigcup_{i \in J} A_{i}$. If $e \in E$ with $\|e\| \leq 1$, then it is easy to see that $\lambda^{-1} \mathcal{X}_{B} e \in \mathcal{V}_{1}$. Therefore, $\|m(B) e\| \leq \varepsilon$ and then $\|m\|\left(X \backslash \bigcup_{i \in J} A_{i} \leq \varepsilon\right.$. This proves $m \in M_{u}(X, \mathcal{L}(E, F))$.

Finally, $T=T_{m}$ on $C_{b}(X, E)$ follows from the $\beta_{u}$-denseness of $C_{r c}(X, E)$ in $C_{b}(X, E)$ and the $\beta_{u}$-continuity of both $T$ and $T_{m}$.

\section{References}

[1] J. Aguayo and J. Sanchez, Weakly compact operators and the strict topologies, Bulletin of the Australian Mathematical Society, Vol. 39, pp. 353-359, (1989).

[2] J. Aguayo, N. De Grande-De Kimpe, S. Navarro, Strict locally convex topologies on $B C(X, \mathbf{K})$, Proc. p-Adic Functional Analysis, Marcel Dekker, Inc., pp. 1 - 9, (1997).

[3] J. Aguayo, N. De Grande-De Kimpe, S. Navarro, Strict topologies and duals in spaces of functions, Proc. p-Adic Functional Analysis, Marcel Dekker, Inc., pp. 1 - 10, (1999). 
[4] J. Diestel and J. Uhl, Vector Measures, Mathematical Surveys and Monographs, Number 15, A.M.S., (1977).

[5] A. Katsaras; Strict topologies in non-archimedean Function Spaces. Internat. J. Math. \& Math. Sci., Vol 1, pp. 23-33, (1984).

[6] A. Katsaras, Integral Representation of Continuous Linear Operators on p-adic Function Spaces, Proc. p-Adic Functional Analysis, Marcel Dekker, Inc., Vol 222, pp. 161-175, (2001).

[7] A.C.M. Van Rooij; Non-archimedean functional analysis Marcel Dekker, (1978).

[8] R.F.Wheeler, A survey of Baire measure y strict topologies, Expo. Math., 2, pp. 97-190, (1983).

\section{José Aguayo}

Departamento de Matemática

Facultad de Ciencias Físicas y Matemática

Universidad de Concepción, Casilla 160-C, Concepción

Chile

e-mail : jaguayo@udec.cl

\section{Elsa Chandía}

Departamento de Matemática

Facultad de Ciencias Físicas y Matemática

Universidad de Concepción,

Casilla 160-C,

Concepción

Chile

e-mail : echandia@udec.cl

and

\section{Jacqueline Ojeda}

Departamento de Matemática

Facultad de Ciencias Físicas y Matemática

Universidad de Concepción,

Casilla 160-C,

Concepción

Chile

e-mail : jojeda@udec.cl 